\title{
Evaluation of the Measles Case-Based Surveillance System in Kaduna State (2010-2012)
}

\author{
Celestine A. Ameh², Muawiyyah B. Sufiyan ${ }^{1}$, Matthew Jacob ${ }^{3}$, Endie Waziri ${ }^{2}$ and \\ Adebola T. Olayinka ${ }^{1}$
}

${ }^{1}$ Ahmadu Bello University Zaria, Abuja, Nigeria; ${ }^{2}$ Nigerian Field Epidemiology and Laboratory Training Program, Abuja, Nigeria; ${ }^{3}$ Kaduna State Primary Health Care Agency, Kaduna, Nigeria

\section{Objective}

To evaluate the case-based Measles surveillance system in Kaduna State of Nigeria and identify gaps in its operations

\section{Introduction}

In Africa, approximately 13 million cases of measles and 650,000 deaths occur annually, with sub-Saharan Africa having the highest morbidity and mortality (1). Measles infection is endemic in Nigeria and has been documented to occur all year round despite high measles routine and supplemental immunisation coverage $(2,3)$. The frequent outbreaks of Measles in Kaduna State prompted the need for the reevaluation of the Measles case-based surveillance system

\section{Methods}

We adapted the updated CDC guidelines on surveillance evaluation to assess the systems usefulness, representativeness, simplicity, timeliness, stability and acceptability. A retrospective record review of the measles case-based surveillance data from 2010-2012 to assess data quality, and representativeness. We calculated the annualized detection rate of measles and non measles febrile rash, proportion of available results, proportion of LGAs that investigated at least one case with blood, proportion of cases that are IgM positive and the incidence of measles. We compared the results with WHO (2004) recommended performance indicators to determine the quality and effectiveness of measles surveillance system

\section{Results}

According to the Stake holders, the case-based surveillance system is still useful and acceptable.The proportion of focal sites reporting declined from $96 \%$ in 2010 to $88 \%$ in 2012 . Median interval between specimen collection and release of result improved from 31 days in 2011 to 16 days in 2012. However the best median turnaround time of 7 days was recorded in 2010. The annualized detection rate of measles and non-measles febrile rash fell below the recommendated WHO standard in 2011 and 2012. Case definitions are simple and understood by all the operators. We found a progressive decline in the timeliness and data quality in the years under review.

\section{Conclusions}

This evaluation showed that the surveillance system was still useful. Also, the efficiency and effectiveness of the laboratory component as captured by the "median interval between specimen collection and the release of results improved in 2012 compared to 2011 and 2010. However, there was a progressive decline in the timeliness and completeness of weekly reports in the years under review

\section{Keywords}

Measles; Case-based; Surveillance; Evaluation; Nigeria

\section{Acknowledgments}

We wish to thank the mangement of Kaduna State Primary Health Care Agency for providing the data needed for the evaluation and the NFELTP for providing the resources

\section{References}

1. Simons E, Ferrari M, Fricks J, Wannemuehler K, Anand A, Burton A, et al. Assessment of the 2010 global measles mortality reduction goal: results from a model of surveillance data. Lancet: 2013, 379(9832):2173-8

2. Onoja AB, Adeniji AJ and Faneye A. Measles complications in a Nigerian hospital setting. Clin Rev Opin. 2013;5(2):18-23.

3. Adu FD, Akinwolere OAO, Uche LN. Low seroconversion rates to measles vaccine among children in Nigeria *. Bull World Health Organ. 1992;70(4):457-60.

\section{${ }^{*}$ Celestine A. Ameh}

E-mail: cameh1085@gmail.com 